\title{
Estudio epidemiológico de exposición a plaguicidas organofosforados y carbamatos en siete departamentos colombianos, 1998-2001
}

\author{
Omayda Cárdenas, Elizabeth Silva, Ligia Morales, Jaime Ortiz \\ Grupo de Salud Ambiental, Instituto Nacional de Salud, Bogotá, D.C., Colombia.
}

Objetivo. Determinar la actividad de la acetilcolineterasa en trabajadores con riesgo de exposición a plaguicidas organofosforados y carbamatos y los plaguicidas más frecuentemente aplicados en los cultivos agrícolas en siete departamentos colombianos durante el período de 1998 a 2001.

Material y métodos. De 1998 a 2001, participaron las entidades territoriales de salud de Boyacá, Caldas, Huila, Meta, Norte de Santander, Santander y Valle del Cauca, con 25.242 trabajadores a quienes se les realizó la determinación de la actividad de la acetilcolinesterasa como biomarcador de exposición a estos plaguicidas mediante el método de Limperos y Ranta modificado por Edson.

Resultados. Del total de trabajadores, el $78,9 \%$ eran hombres y $21,1 \%$ mujeres; el $38,8 \%$ se encontraba en un rango de edad de 26 a 40 años. El 66,1\% reportó pertenecer a un régimen de seguridad social; los oficios con mayor número de trabajadores fueron: fumigador-aplicador con $39,1 \%$ y jornalero con $24,9 \%$. Se realizaron 25.356 pruebas de acetilcolinesterasa, de las cuales, $7,6 \%$ mostraron resultados anormales.

Conclusiones. Con relación a los plaguicidas de importancia en salud pública más usados, se encontraron los organofosforados con $42,4 \%$ seguido por carbamatos con $17,8 \%$, insecticidas organoclorados con $8,4 \%$ y clorinados $6,6 \%$, lo cual hace necesario ampliar el uso de biomarcadores para la vigilancia de trabajadores expuestos a plaguicidas no controlados por el programa.

Palabras clave: plaguicidas, insecticidas organofosforados, carbamatos.

Epidemiological study of organophosphate and carbamate pesticide exposure in 7 separated zones in Colombia

Objective. Acetylcholinesterase activity was measured in workers potentially exposed to pesticides that are frequently used in agriculture in 7 provinces in Colombia between 1998and 2001.

Material and methods. During this period, local health centers in the Departments (provinces) of Boyacá, Caldas, Huila, Meta, Norte de Santander, Santander and Valle del Cauca monitored a total of 25,242 workers for acetylcholinesterase activity. The Limperos and Ranta method, modified by Edson, was used to detect levels of pesticide exposure.

Results. The worker sample consisted of $78.9 \%$ men and $21.1 \%$ women. Thirty-nine percent of the workers were between 26 and 40 years of age, and $66 \%$ had social security. The most common work activities were use of spray applicators (39.1\%) and harvesters $(24.9 \%)$. Of the 25,356 tests for acetylcholinesterase activity, $7.6 \%$ showed abnormal activity levels.

Conclusion. In the zones investigated, organophosphosphates were the most commonly used pesticides $(42.4 \%)$, followed by carbamates $(17.8 \%)$, organochlorines $(8.4 \%)$ and chlorinates (6.6\%). The diversity of pesticides in use underlines the need to increase the variety of biomarkers for monitoring exposed workers.

Key words: pesticides, organophosphorus insecticides, carbamates. 
Los plaguicidas son productos de síntesis donde, además del principio activo, se encuentran impurezas, emulsificantes, solventes y aditivos de toxicidad no despreciable (1-3). Debido a su toxicidad intrínseca, mas no a su selectividad, los plaguicidas pueden poner en riesgo el medio ambiente, organismos no blanco (4) y causar lesiones agudas y crónicas en la salud humana (5-11).

El uso de plaguicidas en Colombia ha seguido los lineamientos generales del mercado de agroquímicos a escala internacional. La producción de plaguicidas en el país en el 2001 fue de 59.530 toneladas métricas sumadas a la importación de 35.460 toneladas métricas de plaguicidas (12). Los organofosforados son los insecticidas más utilizados para el control de plagas en los cultivos tanto a nivel internacional como en nuestro país y, junto con los carbamatos y bipiridilos, son los más frecuentemente involucrados en intoxicaciones en todo el mundo $(3,7,8,10,13,14)$. Entre los insecticidas, la producción de los organofosforados alcanzó las 4.478 toneladas métricas, más las importaciones de 2.031 toneladas métricas de ingrediente activo, materia prima y producto terminado; para los carbamatos, fue de 1.905 toneladas métricas, sumado a las importaciones de 681 toneladas métricas de ingrediente activo y de producto terminado; la producción e importación de organoclorados fue de 782 toneladas métricas (12).

El alto grado de toxicidad de los compuestos organofosforados en los mamíferos, se debe a la fosforilación del ingrediente activo y la consecuente inhibición de la enzima acetilcolinesterasa (AChE), que favorece la desactivación de la acetilcolina en el sistema nervioso. La inhibición de la AChE en sangre, cerebro y otros tejidos causa una excesiva estimulación de los receptores muscarínicos y nicotínicos del sistema nervioso $y$, como consecuencia de la acumulación de la acetilcolina a nivel de sinapsis colinérgica, causa

Correspondencia:

Omayda Cárdenas, Grupo de Salud Ambiental, Avenida Calle 26 No. 51-60, Bogotá, D.C., Colombia. Teléfono: 2207700 , extensiones 447 y 448. ocardenas@ins.gov.co

Recibido: 03/06/04; aceptado: 03/02/05 varios efectos farmacéuticos que culminan con la muerte por paro respiratorio $(5,10,13,15-18)$. Los carbamatos, aunque son de menor persistencia ya que la $A C h E$ se decarbamila rápidamente, también causan inhibición de la AChE; los efectos neurotóxicos son similares a los causados por los organofosforados pero en menor grado con una recuperación usualmente rápida $(14,15,17)$.

La medición de la AChE en eritrocitos o en sangre total y la butirilcolinesterasa en plasma son los biomarcadores desarrollados para evaluar exposición a plaguicidas organofosforados y carbamatos $(15,19,20)$ ya que representan el blanco molecular de la toxicidad de estos plaguicidas. Cuando la enzima es bloqueada no participa en la hidrólisis de la acetilcolina, lo que conlleva una acumulación del neurotransmisor, produciendo efectos tóxicos que involucran los sistemas parasimpático, simpático, motor y nervioso central $(14,15,18)$.

Teniendo en cuenta los problemas de intoxicación por plaguicidas en Colombia, el Ministerio de Salud estableció mediante el Decreto 1843 de 1991, la regulación de las disposiciones sanitarias sobre uso y manejo de plaguicidas y en el artículo 170 determinó que las direcciones seccionales de salud desarrollen un programa específico de vigilancia epidemiológica de plaguicidas conforme a las normas del Ministerio de Salud (21).

El Laboratorio de Salud Ambiental del INS, adelantándose a esta necesidad, viene desarrollando desde 1981 el Programa de Vigilancia Epidemiológica de Plaguicidas Organofosforados y Carbamatos, VEO (22), entre cuyos objetivos está la detección precoz de los casos de intoxicación e impulsar el desarrollo de acciones de promoción, prevención y control que disminuyan la incidencia de estos casos en los trabajadores. Por lo tanto, las seccionales de salud participantes en el programa VEO vienen cumpliendo con esta disposición del Ministerio en lo referente a los organofosforados y carbamatos, que son los plaguicidas que causan mayor número de intoxicaciones en el país $(14,17,23)$.

La información capturada mediante el programa VEO se ha venido publicando desde 1993, obteniéndose datos sobre la frecuencia de 
intoxicación causada por plaguicidas organofosforados y carbamatos, con base en los resultados de los porcentajes de actividad de la AChE en la población con riesgo de exposición ocupacional $(14,24-26)$. Esta información es remitida a las entidades territoriales de salud (ETS), para que se tomen las medidas correctivas, incluso asistencia médica para las personas que presentan porcentajes de actividad de AChE menor o igual a $62,5 \%$.

Con el fin de dar continuidad al reporte de resultados, este estudio presenta el análisis de la información recolectada durante el período de 1998 a 2001 y muestra datos sobre los porcentajes de actividad de AChE en trabajadores con riesgo de exposición a plaguicidas organofosforados y carbamatos y los plaguicidas más frecuentemente aplicados en los cultivos y labores desarrolladas por esta población de trabajadores, información que se empieza a conocer a través de este estudio.

\section{Material y métodos}

\section{Población muestra}

Se realizó un estudio descriptivo de la información reportada al programa VEO durante el período 1998 a 2001, en el que participaron 25.242 trabajadores pertenecientes a siete ETS. Hicieron parte del estudio los trabajadores quienes en el momento de realizar el censo anual en las empresas participantes, estaban ocupacionalmente expuestos a plaguicidas y las personas que se encontraban indirectamente expuestas por habitar en áreas cercanas a las zonas de aplicación e influencia de los plaguicidas.

Antes de iniciar la recolección de la información y de la muestra de sangre, los técnicos de saneamiento visitaron las empresas y fincas e informaron a los trabajadores los objetivos del programa, la importancia de conocer la información suministrada por ellos y el tipo de estudio que se realizaría. Una vez el trabajador acepta participar voluntariamente, se realiza una entrevista y se aplica un formulario para la recolección de la información de variables demográficas, ocupacionales y niveles de actividad de AChE en sangre total; en el caso de niños y menores de edad, la participación se hace con previo consentimiento de los padres.
En el 2000, al formulario de la encuesta se le adicionaron variables relacionadas con exposición, uso y aplicación de plaguicidas, nombre de los plaguicidas más frecuentemente empleados y principales cultivos blanco de fumigación.

\section{Procedimiento de laboratorio}

Para la medición del porcentaje de actividad de AChE, se tomó una muestra de $10 \mu \mathrm{L}$ de sangre, obtenida por punción en el dedo medio con una lanceta, previa desinfección del área de punción. La sangre fue recolectada con una micropipeta y depositada en tubos que contenían solución indicadora (sal sódica de azul de bromotimol soluble en agua); las muestras se mantuvieron a temperatura ambiente máximo por 2 horas en clima cálido o por 4 horas en clima frío, hasta su análisis en campo o en el laboratorio de las ETS.

La determinación de la actividad de AChE se realiza por el método colorimétrico de Limperos y Ranta modificado por Edson (27), empleando el equipo de campo Lovibond, el cual permite determinar el porcentaje de actividad de la enzima en sangre total, de manera rápida, con alta especificidad, precisión y bajo costo (27-30).

El principio del método es el cambio de $\mathrm{pH}$ en el tiempo, detectado como cambio de color y expresado en porcentaje de actividad en valores discretos y a intervalos de $12,5 \%(19,27,30)$. Se consideran anormales los valores iguales 0 menores a $62,5 \%$ de actividad de $A C h E(3,27,30)$. La evaluación del desempeño de los laboratorios de las ETS que reportan los resultados de actividad de AChE, se realiza por medio del Programa Interlaboratorios de Control de Calidad, coordinado por el Grupo Red Salud Ambiental del Instituto Nacional de Salud $(17,22)$.

\section{Análisis de la información}

La información obtenida en la entrevista y los resultados de la actividad de AChE son digitados por cada ETS en un cuestionario previamente diseñado en Epi-Info, por el Grupo Red Salud Ambiental. Una vez registrada la información, es remitida en medio magnético o por correo electrónico al Instituto Nacional de Salud. Utilizando el programa Epi-Info, se hace análisis descriptivo mediante frecuencias simples tanto 
para la población total como para los trabajadores con niveles anormales de porcentajes de actividad de AChE y se realiza estandarización directa para determinar, mediante ajuste de proporciones, las variables con mayor prevalencia de valores anormales de actividad de AChE.

\section{Resultados}

\section{Descripción general y ocupacional}

Durante el período estudiado, participaron en el programa las ETS de Boyacá, Norte de Santander, Meta, Caldas, Huila, Santander y Valle del Cauca (cuadro 1). Del total de trabajadores participantes, $78,9 \%(19.905)$ eran hombres y $21,1 \%$ (5.337) mujeres; el rango de edad con mayor número de trabajadores fue de 26 a 40 años, 38,8\% (9.791), seguido por el de 18 a 25 años, $18,7 \%$ (4.725) tanto para hombres como para mujeres; igualmente, se encontró la participación de personas indirectamente expuestas como niños, $7,8 \%$ (1.964) y mayores de 60 años, $4,1 \%$ (1.034). Del total de participantes, sólo $34,0 \%$ (8.569) pertenecía al régimen de vinculados al Sistema General de Seguridad Social en Salud. Los oficios reportados por los participantes en el momento de realizar la entrevista fueron: fumigador-aplicador, $39,1 \%$ (9.858), seguido por el de jornalero, $24,9 \%$ (6.293), y estudiante, $9,5 \%$ (2.394); las principales actividades laborales desarrolladas por las empresas o fincas fueron: fumigación de cultivos agrícolas, $67,8 \%$ (17.117), distribución o venta de plaguicidas, $9,9 \%$ (2.489) y, 4,7\%, (1.185) agropecuarias.

\section{Exposición y uso de plaguicidas}

Dado que las variables de exposición a plaguicidas fueron adicionadas al formulario en el 2000, la información descrita corresponde al reporte hecho por cinco ETS (una en el 2000 y cuatro en el 2001) de 5.750 trabajadores, de los cuales, $90,0 \%$ (5.176) reportó haber estado expuesto a plaguicidas. El principal uso y aplicación de los plaguicidas, en forma directa o indirecta, fue en labores agrícolas, $73,0 \%(4.179)$, seguido por el uso doméstico, $12,3 \%$ (707), el uso en salud pública para el control de vectores, 8,6\% (497) y en ganadería, 6,1\% (348).

Teniendo en cuenta la clasificación de los plaguicidas según grupo químico, dada por el Ministerio de Salud de Colombia $(21,31)$, de los 8.659 datos reportados por los trabajadores, los más usados fueron los organofosforados, $42,4 \%$ (3.671), seguido por los carbamatos, $17,8 \%$ (1.545), organoclorados, $8,4 \%(730)$, y otros compuestos clorados (clorinados), 6,6\% (574), principalmente. Entre estos plaguicidas, los ingredientes activos más frecuentemente empleados fueron: carbofurán, 15,4\% (1.337), seguido por malatión, $10,5 \%$ (906), endosulfán, $8,4 \%$ (730), y clorpirifos, $7,0 \%$ (607).

Al clasificar los plaguicidas teniendo en cuenta la categoría toxicológica, se encontró que $45,8 \%$ (3.962) pertenecía a la categoría toxicológica I (extremadamente tóxicos), seguida por la categoría III (medianamente tóxicos) con $25,8 \%$ (2.238); en tercer lugar, la categoría toxicológica

Cuadro 1. Trabajadores participantes por año y entidad territorial de salud, 1998-2001.

\begin{tabular}{|c|c|c|c|c|c|c|c|c|c|c|}
\hline \multirow[b]{2}{*}{$\begin{array}{l}\text { Entidad territorial } \\
\text { de salud }\end{array}$} & \multicolumn{2}{|c|}{1998} & \multicolumn{2}{|c|}{1999} & \multicolumn{2}{|c|}{2000} & \multicolumn{2}{|c|}{2001} & \multicolumn{2}{|c|}{ Totales } \\
\hline & $\mathbf{n}$ & $\%$ & $\mathbf{n}$ & $\%$ & $\mathbf{n}$ & $\%$ & $\mathbf{n}$ & $\%$ & $\mathbf{n}$ & $\%$ \\
\hline Boyacá & 857 & 3,4 & 0 & 0,0 & 245 & 1,0 & 0 & 0,0 & 1102 & 4,4 \\
\hline Caldas & 323 & 1,3 & 1103 & 4,4 & 1067 & 4,2 & 1155 & 4,6 & 3648 & 14,5 \\
\hline Huila & 568 & 2,3 & 1152 & 4,6 & 1662 & 6,6 & 490 & 1,9 & 3872 & 15,3 \\
\hline Meta & 563 & 2,2 & 956 & 3,8 & 897 & 3,6 & 0 & 0,0 & 2416 & 9,6 \\
\hline Norte de Santander & 775 & 3,1 & 0 & 0,0 & 1653 & 6,5 & 0 & 0,0 & 2428 & 9,6 \\
\hline Santander & 531 & 2,1 & 1298 & 5,1 & 1717 & 6,8 & 420 & 1,7 & 3966 & 15,7 \\
\hline Valle del Cauca & 3299 & 13,1 & 1099 & 4,4 & 624 & 2,5 & 2788 & 11,0 & 7810 & 30,9 \\
\hline Totales & 6916 & 27,4 & 5608 & 22,2 & 7865 & 31,2 & 4853 & 19,2 & 25242 & 100,0 \\
\hline
\end{tabular}

Fuente: Programa de Vigilancia Epidemiológica de Plaguicidas Organofosforados y Carbamatos (VEO), 1998-2001, Grupo Red de Salud Ambiental, INS. 
II (altamente tóxicos), 25,1\% (2.170), y 3.3\% (289) correspondía a la categoría IV (ligeramente tóxicos). Igualmente, se consideraron según el organismo que se va a controlar encontrando el mayor uso como insecticidas, $77,2 \%$ (6.687), seguido por herbicidas, $13,8 \%(1.197)$ y fungicidas, 8,9\% (772).

Con relación a la aplicación de los plaguicidas en cultivos agrícolas de los 8.917 datos registrados, los principales cultivos en los que se aplicaron los plaguicidas fueron: café, $14,2 \%$ (1.270); granadilla, 11,1\% (989); tomate, $8,7 \%$ (773); en el control de maleza, 8,3\% (736); en el cultivo de maíz, 4,0\% (358), y pimentón, 4,0\% (353), entre otros.

\section{Porcentaje de actividad de la acetilcolinesterasa}

A todos los participantes en el estudio se les determinó el porcentaje de actividad de AChE en sangre, para un total de 25.356 pruebas de AChE. El 7,6\% (1.922) de los trabajadores presentó valores de actividad de AChE menores o iguales a $62,5 \%$, considerados como valores anormales. La figura 1 presenta la distribución por año de los resultados de la actividad de AChE, durante el período 1998-2001.

\section{Trabajadores con porcentajes de actividad de AChE anormales}

\section{Descripción general y ocupacional}

De los 1.922 trabajadores que presentaron pruebas anormales, $78,5 \%$ (1.509) eran hombres y $21,5 \%$ (413) mujeres; el rango de edad con mayor número de trabajadores fue el de 26 a 40 años, $41,5 \%$ (797), seguido por el de 18 a 25 años con $20,9 \%$ (401). Al igual que en la población total participante, se encontró un porcentaje bajo, $29,7 \%$ (571), que pertenecía al régimen de vinculados al Sistema General de Seguridad Social en Salud. Como era de esperarse, los oficios con mayor número de trabajadores con porcentajes de actividad de AChE anormales fueron fumigadoraplicador, 36,4\% (700), seguido por el de jornalero, $29,7 \%$ (571), y estudiante, 10,4\% (199), principalmente, en actividades de fumigación de cultivos agrícolas, 53,5\% (1.028).

\section{Exposición y uso de plaguicidas}

Con relación a las variables de exposición, de los 1.922 trabajadores con pruebas anormales, sólo se obtuvo información en 401 trabajadores, de los cuales, $97,3 \%$ (390) reportó haber estado expuesto a plaguicidas. El uso de los plaguicidas en forma directa o indirecta fue: en actividad agrícola,

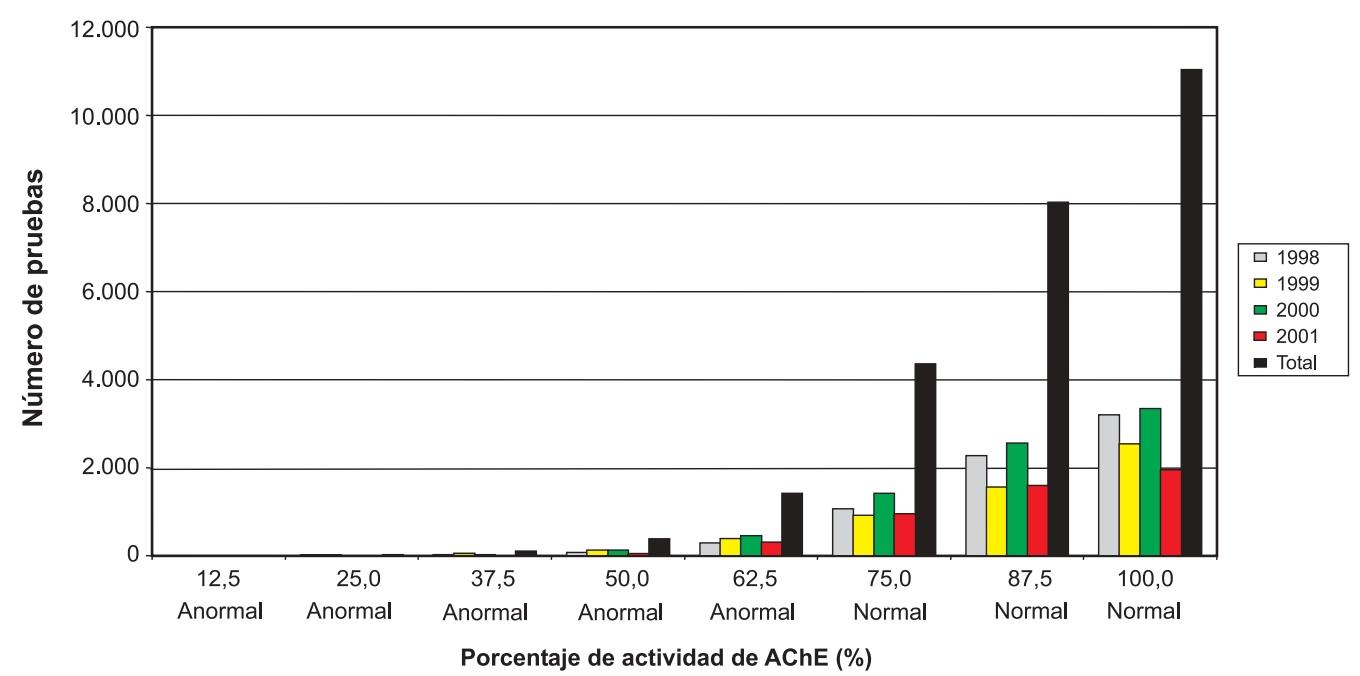

Fuente: Programa de Vigilancia Epidemiológica de Plaguicidas Organofosforados y Carbamatos (VEO) 1993-2001, Grupo Red Salud Ambiental, INS

Figura 1. Distribución de los niveles de actividad de la acetilcolinesterasa (AChE), 1998-2001. 
$86,1 \%$, seguido por el uso doméstico, $9,2 \%$; en salud pública para el control de vectores, $4,4 \%, y$ en ganadería, $0,3 \%$.

La clasificación de los 801 plaguicidas reportados por estos trabajadores, teniendo en cuenta el grupo químico, fue: organofosforados, $46,2 \%$, seguido por los carbamatos, $15,9 \%$, y otros compuestos clorados o clorinados, 9,6\%; por categoría toxicológica, en primer lugar se encontró la categoría I, 43,9\% (352), seguida por la categoría III con 32,6\% (261); categoría II, 19,5\% (156), y categoría IV, 4,0\% (32); el uso de los plaguicidas según organismo que se va a controlar fue: $72,8 \%$ (583) como insecticidas, seguido por herbicidas, $15,2 \%$ (122), y fungicidas, $11,9 \%$ (95). Los principales cultivos en los que se aplicaron los plaguicidas reportados fueron: tomate, $13,3 \%$ (86); pimentón, 9,6\% (62), y en el control de maleza, $8,7 \%(56)$.

\section{Prevalencia de valores anormales en la actividad de AChE}

Al realizar el ajuste de proporciones mediante estandarización directa, se encontró que la ETS que reportó mayor número de trabajadores con pruebas anormales en el período de 1998-2001 fue la de Santander, 19,6\%, seguida por Huila, $9,6 \%$, y en tercer lugar, Meta, 7,5\%; igualmente, se hizo la estandarización directa de proporciones con el fin de realizar una comparación de pruebas anormales de AChE durante los años en los que se han reportado datos del VEO (1993 a 2001) y se encontró que la mayor frecuencia de valores anormales de actividad de AChE fue en 1999 (figura 2).

Al comparar mediante estandarización directa, la prevalencia de valores anormales de actividad de AChE según el oficio desempeñado y teniendo en cuenta la población total de 95.599 trabajadores (1993 a 2001), el mayor riesgo de intoxicación se encontró en el oficio de mezclador-tanqueador seguido por el de banderero y, en tercer lugar, el de fumigador-aplicador. Para el período de 19982001, aunque los oficios reportados con mayor número de trabajadores tanto en la población total como en los que presentaron porcentajes de actividad de AChE anormal fueron: fumigadoraplicador, jornalero y estudiante; al hacer el ajuste

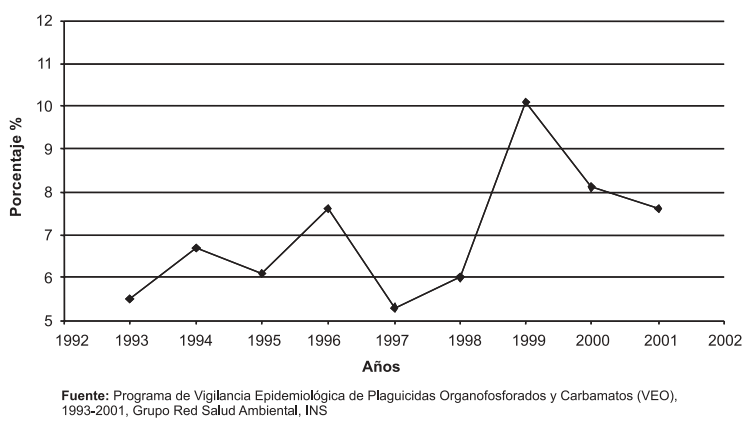

Figura 2. Tasas estandarizadas de anormalidad de la actividad de la acetilcolinesterasa (AChE), 1993-2001.

de proporciones mediante estandarización directa, se encontró que los oficios con mayor riesgo fueron los de mezclador-tanqueador, servicios generales de campo y jornalero (cuadro 2 ).

\section{Discusión}

Las intoxicaciones por plaguicidas constituyen un capítulo importante en la historia ya que han ocurrido graves hechos en todo el mundo, donde estos compuestos químicos han causado enfermedades y muerte en grandes poblaciones. En Colombia, el uso intensivo e indiscriminado de insumos agrícolas, especialmente de organofosforados y carbamatos, viene generando innumerables problemas sobre la salud de la población, como ha sido reportado en los estudios del programa VEO $(14,24-26)$ y en algunos casos de intoxicación registrados en el país $(17,23)$, datos que coinciden con lo reportado a nivel internacional donde las intoxicaciones son causadas principalmente por organofosforados, seguido por carbamatos, organoclorados, compuestos mercuriales orgánicos y los bipiridilos (específicamente, paraquat) $(6-8,32)$.

Por lo anteriormente expuesto y por lo encontrado en el presente estudio, es indispensable mantener una vigilancia constante en el uso de este tipo de plaguicidas; sin embargo, la participación de las ETS y el reporte de información al programa VEO es bajo en comparación con los años anteriores $(14,24)$. Esto puede ser debido a problemas de orden público y a las políticas de descentralización administrativa que han obstaculizado la consolidación de una verdadera red nacional de 
Cuadro 2. Comparación de prevalencias de anormalidad de la actividad de la acetilcolinesterasa (AChE), según oficio, 1993-2001.

\begin{tabular}{lccc}
\hline Oficios & \multicolumn{2}{c}{ Porcentaje de anormalidad de AChE } \\
\cline { 2 - 4 } & $\mathbf{1 9 9 3 - 1 9 9 7}$ & $\mathbf{1 9 9 8 - 2 0 0 1}$ & $\mathbf{1 9 9 3 - 2 0 0 1}$ \\
\hline Vendedor & $4,2(49 / 1.162)$ & $5,1(41 / 804)$ & $4,6(90 / 1.966)$ \\
Banderero & $14,6(7 / 48)$ & $5,6(5 / 90)$ & $8,7(12 / 138)$ \\
Almacenista-bodeguero & $7,2(101 / 1.411)$ & $6,0(30 / 503)$ & $6,8(131 / 1.914)$ \\
Mezclador-tanqueador-formulador-equipador & $17,7(73 / 412)$ & $16,4(28 / 171)$ & $17,3(101 / 583)$ \\
Fumigador-aplicador & $7,8(1489 / 19.066)$ & $7,1(700 / 9.858)$ & $7,6(2.189 / 28.924)$ \\
Piloto-aviador & $10,3(8 / 78)$ & $4,9(13 / 265)$ & $6,1(21 / 343)$ \\
Conductor & $3,8(24 / 628)$ & $4,3(11 / 254)$ & $4,0(35 / 882)$ \\
Servicios generales del campo & $4,3(52 / 1.222)$ & $9,9(91 / 916)$ & $6,7(143 / 2.138)$ \\
Agrónomo-profesiones afines & $4,8(88 / 1824)$ & $5,2(11 / 213)$ & $4,9(99 / 2.037)$ \\
Jornalero & $6,0(1.042 / 17.432)$ & $9,1(571 / 6.293)$ & $6,8(1613 / 23.725)$ \\
Profesor-educador-instructor & $4,6(9 / 197)$ & $6,0(6 / 100)$ & $5,1(15 / 297)$ \\
Estudiante-escolar & $5,5(417 / 7.593)$ & $8,3(199 / 2.394)$ & $6,2(616 / 9.987)$ \\
Ama de casa-niños-residentes mayores 60 años & $4,0(181 / 4.558)$ & $7,9(133 / 1.684)$ & $5,0(314 / 6.242)$ \\
Profesionales de diferentes ramas & $8,7(21 / 241)$ & $3,9(10 / 258)$ & $6,2(31 / 499)$ \\
Administrativos & $5,4(751 / 13961)$ & $5,1(73 / 1.439)$ & $5,4(824 / 15.400)$ \\
Otros & $4,8(25 / 524)$ & 0 & $4,8(25 / 524)$ \\
\hline
\end{tabular}

Fuente: Programa de Vigilancia Epidemiológica de Plaguicidas Organofosforados y Carbamatos (VEO), 1993-2001, Grupo Red Salud Ambiental, INS.

laboratorios de vigilancia epidemiológica, impidiendo tener mayor información sobre la situación nacional relacionada con el uso de plaguicidas organofosforados y carbamatos.

No obstante, la información obtenida es muy valiosa y nos permite tener una idea global sobre el tipo de plaguicidas empleados en cinco departamentos del país, en los cuales se pueden realizar intervenciones específicas en cambios conductuales relacionados con el uso y manejo de los plaguicidas de una manera más segura, a nivel de la empresa, de los trabajadores y de los miembros de su familia.

Con relación al sexo, se observó que tanto hombres como mujeres poseen el mismo riesgo de exposición, ya que la proporción de valores anormales de actividad de AChE para hombres fue de $1: 13,2$ y para mujeres de $1: 12,9$, lo cual refuerza el planteamiento de procurar una mejoría de las condiciones de higiene y seguridad en las labores cotidianas para los trabajadores y sus familias, ofreciendo campañas educativas de capacitación y sensibilización en el uso y manejo seguro de los plaguicidas.

Respecto a la edad, el mayor porcentaje de valores anormales de actividad de AChE se encontró en los grupos de 18 a 40 años (62,4\%) que incluía los dos sexos, lo cual es debido a que los trabajadores que se encuentran en estos rangos de edad son empleados en oficios de alto riesgo $(73,9 \%)$. También es importante resaltar la presencia de niños menores de 11 años $(8,7 \%)$ y de personas mayores de 60 años $(3,4 \%)$, con valores anormales de actividad de AChE, frecuencias que son mayores que las de años anteriores $(14,25)$.

Según la información dada por algunas ETS, las intoxicaciones en esta población, probablemente, se presentan por el incumplimiento de las normas de seguridad, por la exposición indirecta al encontrarse en los alrededores de las áreas donde se aplican los plaguicidas y por el consumo de alimentos sin las medidas adecuadas de higiene.

Con relación a la información reportada por oficios, se encontró un alto porcentaje de estudiantes, amas de casa, niños menores de 5 años-personas mayores de 60 años, con valores de actividad de AChE menores o iguales a $62,5 \%$. Estas personas conforman un grupo vulnerable de alto riesgo no laboral y, según información de las ETS, se debe a que ayudan en labores de campo manipulando directamente los plaguicidas pero sin 
vinculación directa con la empresa, sus viviendas e instituciones educativas se encuentran en áreas cercanas a los sitios de fumigación tanto agrícola como de campañas sanitarias, consumen alimentos con residuos de plaguicidas o tienen contacto con ropa y elementos contaminados.

Al realizar la estandarización directa de las tasas de anormalidades de actividad de AChE durante el período de 1993 a 2001, se encontró que los oficios de mezclador-tanqueador, banderero y fumigador-aplicador, presentaban mayor frecuencia de intoxicación por organofosforados y carbamatos, lo cual no es de extrañar ya que las personas empleadas en estas labores tienen exposición directa a agroquímicos; además, muchas de ellas utilizan de manera deficiente los elementos de protección personal o no los usan por condiciones climáticas y topográficas o por desconocimiento del riesgo al que están expuestas. La misma situación es válida para los oficios de mezclador-tanqueador, servicios generales de campo y jornalero que, en el presente estudio, fueron los que presentaron mayor frecuencia de anormalidades de actividad de AChE después de aplicar la estandarización directa.

Al analizar las tasas de anormalidades de actividad de AChE desde 1993, se encontró un aumento considerable de valores anormales en 1998 y 1999 ; este hecho podría deberse, según información suministrada por las ETS, a la disminución en el control y capacitación a los trabajadores con respecto al uso y el manejo de los plaguicidas, debido a problemas de orden público y a recortes presupuestales. Aunque en el 2000 y 2001 se encontró una leve disminución de las tasas de anormalidades de actividad de AChE, no es significativa, considerando la poca cobertura registrada durante este período y el sesgo en la muestra de la población ya que es obtenida por un proceso de autoselección; de forma similar, por la dificultad de acceso a sitios apartados, la mayoría de los participantes laboraban en lugares cercanos a las cabeceras municipales que es donde tienen mayor posibilidad de recibir capacitación en el uso y manejo de plaguicidas. La frecuencia de anormalidades de actividad de AChE para el 2001 fue de $7,6 \%$, porcentaje levemente menor comparado con los dos años anteriores y similar al reportado en estudios internacionales $(29,33)$. No obstante, no es una frecuencia representativa del territorio nacional, considerando la baja participación de las ETS durante el período estudiado.

Con relación al uso de plaguicidas, es importante resaltar el alto porcentaje $(71,0 \%)$ de aplicación de insecticidas categoría toxicológica I y II (extremadamente tóxicos y altamente tóxicos), respectivamente, y entre éstos los principales grupos químicos usados los organofosforados, los carbamatos y los organoclorados. Éstos son plaguicidas de amplio espectro que, al igual que en otros países, son introducidos a la agricultura $y$ en los lugares de trabajo causando enfermedades entre los trabajadores que manipulan, mezclan y aplican estos compuestos y en personas que están en los alrededores de los campos y cultivos tratados, ya que son fácilmente absorbidos a través de la piel y por la vía respiratoria como principales rutas de exposición $(3,5,28)$.

Aunque el objetivo del programa VEO es la vigilancia de plaguicidas organofosforados y carbamatos, es importante resaltar la información obtenida en el presente estudio sobre el uso de grupos químicos como los organoclorados, otros compuestos clorados o clorinados y bipiridilos, algunos de los cuales han sido prohibidos 0 restringidos en nuestro país, según las condiciones de uso, por los posibles efectos en la salud del hombre y el medio ambiente.

Entre estos plaguicidas se encuentra el endosulfán $(8,4 \%)$, de uso restringido sólo para el control de la broca en el café desde 1997 (34) y prohibido definitivamente en el 2001 (35); igualmente, se encontró el uso de bipiridilos, principalmente, el paraquat $(5,7 \%)$, plaguicida de uso restringido (34).

Aunque con porcentaje bajo, también se encontró el uso de plaguicidas como alaclor, atrazina, benomil, diclorvos, captán, dimetoato y clorotalonil, muchos de ellos restringidos por la EPA a comienzos de los años 90 por ser clasificados por esta agencia como prioritarios por el potencial carcinogénico que poseen y por producir riesgos agudos en salud $(1,3,36)$. El uso de estos plaguicidas en nuestro país podría ser explicado 
por las existencias de la producción e importación de producto terminado hasta el 2001 (12).

Entre las limitaciones del estudio se encontró, principalmente, que no se cuenta con información sobre las cantidades aplicadas de plaguicidas, el diligenciamiento incompleto de los formatos de encuesta, la baja participación de las ETS, la falta de continuidad de los departamentos en el programa, los problemas administrativos por cambio o remoción de los funcionarios responsables del programa y la sensibilización traducida en el apoyo de las directivas en cada una e las ETS. Igualmente, se tiene el sesgo de información que no es capturada en el formulario de encuesta y es suministrada por algunas ETS, sobre causas probables de los resultados obtenidos y, aunque sólo es apreciativa, la hemos tenido en cuenta en la discusión con el objeto de plantear hipótesis que conduzcan al fortalecimiento del programa VEO y al planteamiento de proyectos de investigación.

Con relación a los resultados de los valores de la actividad AChE, se observó un alto porcentaje de valores normales en los trabajadores participantes. Sin embargo, hasta el 2001, es limitada la interpretación de las diferencias de valores de actividad AChE, considerando que en la información obtenida en este estudio, no se tuvo una línea base de preexposición, niveles de control y seguimiento posexposición en los trabajadores.

Cuando se sospecha la exposición a organofosforados y carbamatos, un resultado dentro de los rangos normales de actividad, y en ausencia de valores de AChE basales, no es suficiente para diagnosticar ausencia de intoxicación $(9,18,28)$.

En nuestro país, aún no se dispone de cifras exactas y confiables sobre las intoxicaciones humanas relacionadas con el uso de plaguicidas. Esto se podría deber a una falta de mecanismos administrativos adecuados, a la poca o ninguna información confiable relacionada con el registro de casos de intoxicación por plaguicidas, a la deficiencia de laboratorios para realizar pruebas confirmatorias, a que el trabajador con valores anormales de actividad de AChE no busca atención en los servicios de salud por desconocimiento de los signos y síntomas, temor a perder el empleo o porque muchas veces se encuentra en comunidades que tienen dificultad para acceder a los servicios de salud 0 , simplemente, no cuentan con ningún tipo de vinculación al Sistema General de Seguridad Social en Salud.

Con base en las limitaciones citadas, se creó la necesidad de ajustar e implementar el formato de encuesta y mejorar los sistemas para obtener mejor calidad en la recolección de la información a nivel nacional, mayor utilidad en los datos reportados sobre el tiempo de exposición a los plaguicidas, reporte de plaguicidas más usados y niveles basales de actividad de AChE, de control y seguimiento en los trabajadores expuestos y capacitar al personal responsable del programa en cada una de las ETS. Es por esto que en el 2003 el Grupo Red Salud Ambiental capacitó 28 funcionarios de las diferentes ETS del país en la recolección de la información y el diligenciamiento del nuevo formato de encuesta y se adquirieron compromisos para fortalecer la participación y ampliar la cobertura a nivel nacional.

\section{Recomendaciones}

Con base en lo encontrado en este estudio, se hace necesario fortalecer el programa de vigilancia, mejorar los sistemas de recolección de información, capacitar al personal responsable de la recolección de la información y análisis de muestras en el laboratorio, recuperar la participación y ampliar la cobertura a otras ETS en todo el país, con el propósito de tener un panorama general sobre la verdadera situación a nivel nacional relacionada con los principales plaguicidas que están causando intoxicaciones y así tomar medidas de prevención y control que nos ayuden a minimizar la prevalencia de estos casos de intoxicación.

Se hace necesario un esfuerzo conjunto de organismos de salud, entidades de salud y educativas con el fin de desarrollar programas de capacitación y educación en el uso y manejo seguro de los plaguicidas a los trabajadores y población general, con el fin de prevenir y controlar los casos de intoxicación y así proteger la salud de los trabajadores y miembros de sus familias.

Así mismo, teniendo en cuenta que el biomarcador empleado $(\mathrm{AChE})$ en el programa VEO, determina 
exposición específica a organofosforados y carbamatos, se hace necesario evaluar la exposición a otros plaguicidas de importancia en salud pública como los organoclorados y clorinados, considerando su porcentaje de uso, lo cual amerita fortalecer y ampliar el programa de vigilancia, estandarizando nuevas metodologías de laboratorio sensibles, rápidas y de bajo costo como la cromatografía en capa fina, entre otras, que permita monitorear y evaluar exposición a estos tóxicos.

La problemática descrita no sólo afecta al trabajador agrícola que sufre la mayor exposición a los plaguicidas, sino también al medio ambiente en general $y$, por consiguiente, a la comunidad que se ve afectada por la contaminación de alimentos, aguas, suelos y aire, lo cual hace necesario aunar esfuerzos con otros organismos de salud, entidades de salud pública y protección ambiental para fortalecer la vigilancia ambiental y realizar estudios de residuos de plaguicidas en alimentos, aguas, aire y suelos. Igualmente, desarrollar e implementar estrategias que conduzcan a una significativa reducción del uso de plaguicidas sintéticos, el desarrollo de alternativas menos tóxicas como el Manejo Integrado de Plagas (MIP) y agricultura orgánica para proteger la salud humana y el ambiente $(8,32)$.

\section{Agradecimientos}

Los autores agradecen a los trabajadores, gerentes de empresas y propietarios de haciendas o fincas participantes en el Programa de Vigilancia Epidemiológica de Plaguicidas Organofosforados y Carbamatos, VEO; a los responsables del programa Judy Ibañez de Boyacá, Gloria Rivera de Huila, María Leonor Díaz de Meta, Gladys Mora de Norte de Santander, Sandra Bayona de Santander, Eduardo Antonio Vargas de Caldas e Iván Duque y Libardo Cuellar de Valle del Cauca e, igualmente, a los promotores de salud, técnicos de saneamiento básico y personal a cargo del programa VEO en cada ETS por el apoyo logístico para la recolección y la digitación de la información.

\section{Conflicto de intereses}

Los autores manifiestan que no existe ningún conflicto de intereses.
El estudio corresponde al análisis de la base de datos que son reportados por las entidades territoriales de salud que participan en el Programa de Vigilancia Epidemiológica de Plaguicidas Organofosforados y Carbamatos, VEO, liderado por el Grupo Red Salud Ambiental, por medio de convenios interadministrativos de cooperación científica y tecnológica.

\section{Financiación}

Las fuentes de financiación de las ETS son del Plan de Atención Básico (PAB) y, por parte del Instituto Nacional de Salud, son recursos asignados al Grupo Red Salud Ambiental de la Subdirección Red Nacional de Laboratorios.

\section{Referencias}

1. Rodríguez CA. Plaguicidas, necesidad y posibilidades de limitar su uso. Jornadas interdisciplinarias y tripartitas agro: "trabajo y salud". http://www.cepis.org.pe/ eswww/ocupfile/plaguicidas.doc (en línea). [11 de agosto de 2000]; disponible en www.cepis.org.pe. Agosto de 1999; p.1-17.

2. Petrelli G, Siepi G, Miligi L, Vineis. Solvents in pesticides. Scand J Work Environ Health 1993;19:63-5.

3. Henao S, Corey G. Plaguicidas inhibidores de las colinesterasas. Serie de Vigilancia 11. Metepec, México: Asistencia Editorial de Clemente Aguilar; 1991. p.17169.

4. Auteri D, Mammone T. Pestigis: sistema de decisión de apoyo para la valoración del impacto ambiental de plaguicidas en Lombardia, Italia. Boletín Técnico 1999;2:2-7.

5. al-Saleh IA. Pesticides: a review article. J Environ Pathol Toxicol Oncol 1994;13:151-61.

6. Palacios ME, Paz P, Hernández S, Mendoza L. Sintomatología persistente en trabajadores industrialmente expuestos a plaguicidas organo-fosforados. Salud Pública Mex 1999;41:55-61.

7. Visentin S, Fait A. EI proyecto IPCS/OMS sobre vigilancia epidemiológica en las intoxicaciones agudas por plaguicidas. Boletín Técnico 2002;5:1.

8. García JE. Intoxicaciones agudas con plaguicidas: costos humanos y económicos. Rev Panam Salud Pública 1998;4:383-7.

9. López-Carrillo L, López-Cervantes M. Effect of exposure to organophosphate pesticides on serum cholinesterase levels. Arch Environ Health 1993;48:35963.

10. Weisenburger DD. Human health effects of agrichemical use. Hum Pathol 1993;24:571-6 
11. Sharara FI, Seifer DB, Flaws JA. Environmental toxicants and female reproduction. Fertil Steril 1998; 70:613-22.

12. Ministerio de Agricultura y Desarrollo Rural, Instituto Colombiano Agropecuario. Comercialización de plaguicidas 2000-2001: producción-ventas, importación-exportación. Bogotá: Editorial Produmedios; 2003. p.8-124.

13. Dewer YM. Intoxicaciones por organofosforados y agentes terapéuticos: perfil clínico. Boletín Técnico 2002;5:3

14. Silva E, Morales L, Ortiz J. Evaluación epidemiológica de plaguicidas inhibidores de acetilcolinesterasa en Colombia, 1996-1997. Biomédica 2000;20:200-9.

15. Lotti M. Cholinesterase inhibition: complexities in interpretation. Clin Chem 1995;41:1814-8.

16. Zejda JE, McDuffie HH, Dosman JA. Epidemiology of health and safety risks in agriculture and related industries practical applications for rural physicians. West J Med 1993;158:56-63.

17. Toro G, Cárdenas O, Varona M. Neurotoxicidad (III parte): plaguicidas. Acta Neurol Colomb 2002;18:32-50.

18. O'Malley M. Clinical evaluation of pesticide exposure and poisonings. Lancet 1997;349:1161-6.

19. Carmona J, Henao S, Garcés R. Valores de referencia de actividad colinesterásica sanguínea en población laboral activa no expuesta a plaguicidas inhibidores de colinesterasa. Revista Facultad Nacional de Salud Pública 2000;18:55-72.

20. Carmona J. Valores de referencia de colinesterasa plasmática con los métodos de Michel, EQM® y Monotest $\circledast$ en población laboral activa del departamento de Antioquia, Colombia. Biomédica 2003;23:437-55.

21. Ministerio de Salud. Uso y manejo de plaguicidas. Decreto Número 1843 de 1991. Bogotá, 1991:5-47

22. Cárdenas O, Ortiz J, Varona M, Morales L, Acosta H. Algunas consideraciones sobre plaguicidas y estudios realizados en el Laboratorio Salud Ambiental. En: Toro G, Hernández CA, Raad J, editores. Instituto Nacional de Salud 1917-1997: una historia, un Compromiso. Bogotá: Instituto Nacional de Salud; 1998. p.379-89.

23. Idrovo AJ. Intoxicaciones masivas con plaguicidas en Colombia. Biomédica 1999;19:67-76.

24. Varona M, Morales L, Ortiz J, Sánchez JF, Cárdenas O, De la Hoz F. Panorama epidemiológico de exposición a plaguicidas inhibidores de colinesterasa en 17 departamentos del país. Biomédica 1998;18:22-9.

25. Silva E, Morales L, Ortiz JE. Evaluación epidemiológica de plaguicidas en el departamento del Meta, Programa VEO, 1996 a 1997. Inf Quinc Epidemiol Nac 2002;7:389401.

26. Morales L, Silva E, Ramírez P, García A, Ortiz J. Intoxicación por plaguicidas en el departamento del Valle del Cauca. Inf Quinc Epidemiol Nac 1998;3:2224.

27. Edson EF, Fenwick ML. Measurement of cholinesterase activity of whole blood. Brit Med J 1955;1: 1218.

28. Wilson BW, Sanborn JR, O'Malley MO, Henderson JD, Billitti JR. Monitoring the pesticide-exposed worker. Occupational Medicine. Seattle, Washington: Editorial Harborview Medical Center; 1998. p.347-363.

29. Fillmore CM, Lessenger JE. A cholinesterase testing program for pesticide applicators. J Occup Med 1993; 35:61-70.

30. Limperos G, Ranta KE. A rapid screening test for the determination of the approximate cholinesterase activity of human blood. Science 1953;117:453-55.

31. Ministerio de Salud. Grupo de Protección de la Salud. Bogotá, D.C.: Ministerior de la Salud; 2001.

32. Keifer M, Rivas F, Moon JD, Checkoway $H$. Symptoms and cholinesterase activity among rural residents living near cotton fields in Nicaragua. Occup Environ Med 1996;53:726-9

33. Ames RG, Brown SK, Mengle DC, Kahn E, Stratton J, Jackson R. Cholinesterase activity depression among California agricultural pesticide applicators. Am $\mathrm{J}$ Ind Med 1989;15:143-50

34. Ministerio de Salud, Ministerio del Medio Ambiente, Ministerio de Trabajo y Seguridad Social, Ministerio de Agricultura y Desarrollo Rural. Perfil Nacional para una gestión racional de las sustancias químicas. Serie de investigaciones. Santa Fe de Bogotá: Editorial Gente Nueva Ltda.; 1998. p.38-40.

35. Consejo de Estado. Endosulfán sí es peligroso y está prohibido en Colombia. Comunicado para publicación abril 2 de 2001, Bogotá, D.C.; 2001.

36. Woodruff TJ, Kyle AD, Bois FY. Evaluating health risks from occupational exposure to pesticides and the regulatory response. Environ Health Perspect 1994;102: 1088-96. 